\title{
Intentional and incidental encoding of item and associative information in the directed forgetting procedure
}

\author{
William E. Hockley $^{1} \cdot$ Fahad N. Ahmad ${ }^{1} \cdot$ Rosemary Nicholson $^{1}$
}

Published online: 25 September 2015

(C) Psychonomic Society, Inc. 2015

\begin{abstract}
The intentional and incidental encoding of individual words and associations between pairs of words was examined using the item-based directed forgetting procedure. Item and associative recognition were both greater for word pairs followed by a remember $(\mathrm{R})$ cue than a forget $(\mathrm{F})$ cue. Associative discrimination for F-cued pairs was above chance in most conditions, demonstrating that relational informational is encoded incidentally. Item, but not associative, discrimination increased with longer presentation time prior to the cue, indicating that the encoding of item information benefited from maintenance rehearsal but the encoding of relational information did not (Experiments $1 \mathrm{~A}$ and $1 \mathrm{~B})$. The incidental encoding of associations was, though, greater for pairs of words with pre-experimental associations (e.g., needle point), but these pre-experimental associations did not improve memory for pairs that participants tried to remember (Experiment 2). This pattern of results for R- and F-cued pairs mimicked the age-related associative deficit observed by Ahmad, Fernandes, and Hockley (Aging, Neuropsychology and Cognition, 22, 452-472 2015) in comparisons of associative memory between young and older adults.
\end{abstract}

Keywords Associative recognition · Item recognition · Item-based directed forgetting - Compound word effect . Incidental encoding $\cdot$ Maintenance rehearsal

Memory involves representations of individual events (item information) and relations between those events (associative

William E. Hockley

whockley@wlu.ca

1 Department of Psychology, Wilfrid Laurier University, Waterloo, ON N2L 3C5, Canada information) (Murdock, 1974). Forming new associations is a fundamental aspect of building and expanding knowledge structures. Associations between individual items and between items and their contexts also play a critical role in the retrieval of stored information. Therefore, the encoding and retrieval of new associations is an essential aspect of successful memory.

The focus of the present study concerns intentional and incidental encoding of item and associative information. In typical laboratory studies of associative memory, participants are presented with pairs of items and are instructed to remember that each pair occurred together in the study list. Memory for associative information is therefore intentional and strategic. In everyday life, however, relational information is often encoded incidentally, or without any deliberate attempt to do so. For example, associations between attended information and the environmental or situational context are typically encoded incidentally, but such associations can influence later memory of the attended information (e.g., Godden \& Baddeley, 1975; Hockley, 2008). The present study was designed to compare incidental encoding with intentional encoding of item and associative information.

An early examination of incidental and intentional encoding of item and associative information was carried out by Hockley and Cristi (1996). In this study, participants were instructed either to remember only the individual words or to remember an association between the words of each study pair. Immediate tests examined memory for the instructed information and reinforced the need to encode only the instructed information. A final yes-no recognition test examined memory for both item and associative information. In the item recognition test, participants discriminated between old words presented in the previous study list and new words. The associative recognition test involved the discrimination of intact or studied pairs and rearranged pairs of items that had been studied in different pairs. Item information cannot assist in this discrimination as both intact (old) and rearranged (new) pairs consist of old items. Associative recognition, therefore, 
provides a relatively pure test of memory for relational information (Humphreys, 1976).

Hockley and Cristi (1996) found that participants could emphasize the encoding of item information at the expense of associative information, but not the reverse. That is, recognition memory for the individual items was similar in the two encoding conditions. Although associative recognition accuracy was significantly attenuated when participants emphasized the encoding of item information, discrimination of intact and rearranged test pairs was still above chance. Thus, associative information was encoded incidentally or unintentionally, although not to the same extent as when forming associations between pairs of items was the focus of study.

Jou (2010) presented further evidence that participants encode associative information even when the task does not require them to do so. Participants were shown pairs of unrelated words and the instructions "emphasized that the subjects needed only to memorize the two words, but not the pairing or association of the two words" (p. 779). Jou found that pair recognition, where participants indicate whether or not both words of each test pair are studied items, was significantly greater and response time was faster for intact compared to rearranged pairs of studied items demonstrating an influence of associative information on item recognition performance.

Finally, Bancroft, Hockley and Farquhar (2013), examined item-based directed forgetting for associative information. In this procedure, study items are followed by a cue that indicates whether participants should try to remember or forget each item, or in this experiment, pair of items. Bancroft et al. found that associative recognition was greater for pairs that were cued to be remembered compared to the pairs that were cued to be forgotten (i.e., a directed forgetting effect). Although not the main purpose of their study, Bancroft et al. also found that associative recognition for the to-be-forgotten pairs was still above chance demonstrating the incidental encoding of associative information.

The item-based directed forgetting procedure would appear to be an ideal paradigm with which to examine intentional and incidental memory for associations between items. The commonly accepted explanation of item-based directed forgetting is selective rehearsal (Basden \& Basden, 1998; Basden, Basden \& Gargano, 1993, Bjork, 1989; see Golding \& Long, 1998; Johnson, 1994; and MacLeod, 1998, for reviews). Items are initially maintained in short-term or working memory until the remember $(\mathrm{R})$ or forget $(\mathrm{F})$ cue is given. Items followed by an $\mathrm{R}$ cue then receive elaborate rehearsal whereas items followed by an F cue are not rehearsed. As H. M. Johnson (1994) has argued, the item-based directed forgetting procedure is not truly directed forgetting, but rather a manipulation of intention to learn. In this view, the principal effect of $R$ and $F$ cues can be considered one of intentional versus incidental learning.
The item-based directed forgetting procedure has an additional benefit for the examination of incidental memory for associations. In contrast to the studies of Hockley and Cristi (1996) and Jou (2010), where participants were instructed to remember individual words but not their pairing, in the directed forgetting procedure participants are instructed to disregard both the individual items and their association. Therefore, participants have no incentive whatsoever to remember relations between items of pairs that are cued to be forgotten.

The primary purpose of the present study was to use the item-based directed forgetting procedure to examine intentional and incidental encoding of both item and associative information. Yes-no item and associative recognition were used to assess memory for each type of information.

In the directed-forgetting experiment for associative information reported by Bancroft et al. (2013), the pairs of items were presented for $3 \mathrm{~s}$ before the ensuing $\mathrm{R}$ or $\mathrm{F}$ cue was presented. Ideally, participants should simply have held the two words in mind for $3 \mathrm{~s}$ and not deliberately generate any associations between them until an R cue is presented. It is possible, however, that participants could not help thinking of an association, or attempted to anticipate $\mathrm{R}$ cues, while waiting $3 \mathrm{~s}$ for the cue. A second purpose of the present study was to reduce the duration pairs were shown before the cue was presented in order to reduce the time participants had to deliberately or spontaneously generate associations between the items. In Experiment 1A pairs were presented for $2 \mathrm{~s}$ before the presentation of the cue and in Experiment 1B this interval was reduced to $1 \mathrm{~s}$. A comparison of Experiments 1A and 1B, therefore, also permitted an assessment of the effect of the duration of maintenance rehearsal on memory for item and associative information. Finally, in Experiment 2, the ease of generating associations between items in $\mathrm{R}$ and $\mathrm{F}$ cued pairs was manipulated by comparing pairs of words that had pre-experimental associations with randomly paired words.

\section{Experiments 1A and 1B}

In Experiments 1A and 1B, both yes-no item and associative recognition were examined for random word pairs. Pairs were initially presented for $2 \mathrm{~s}$ in Experiment $1 \mathrm{~A}$ and $1 \mathrm{~s}$ in Experiment $1 \mathrm{~B}$ before the $\mathrm{R}$ or $\mathrm{F}$ cue was shown. It was anticipated that item recognition might derive a benefit from maintenance rehearsal during this interval as previous research has demonstrated modest benefits of maintenance rehearsal for item information (Glenberg \& Adams, 1978; Naveh-Benjamin \& Jonides, 1984). Woodward, Bjork and Jongeward (1973) varied the maintenance rehearsal period for single words before the cue from 0 to $12 \mathrm{~s}$ in the item-based directed forgetting procedure. They found that maintenance rehearsal did not aid recall whereas recognition 
increased systematically with rehearsal duration. Their results suggest that maintenance rehearsal strengthened memory for the individual items, but not memory for associations between items, or between items and their context, that would improve recall performance. Based on these results, we predicted that item recognition would benefit from the greater maintenance rehearsal interval of Experiment 1A compared to Experiment 1B. Predictions regarding the benefits of maintenance rehearsal on associative recognition were less clear. It has been argued that free recall performance is based on associations between items and their spatiotemporal context whereas associative recognition depends to a greater extent on item-to-item associations, and therefore these tasks could react differently in many experimental manipulations (e.g., Hanczakowski, Pasek, \& Zawadzka, 2012).

\section{Method}

Participants All participants in each experiment were undergraduate students at Wilfrid Laurier University who volunteered to participate in return for credit in a junior psychology course. There were 24 different students in each of Experiments $1 \mathrm{~A}$ and $1 \mathrm{~B}$.

Apparatus and materials Stimulus presentation and response recording were controlled by SuperLab 3.0 software (Cedrus Corp., San Pedro, CA, USA) run on IBM-compatible PCs with 17-in LCD monitors housed in individual cubicles. The word pool consisted of 176 nouns selected from the MRC Psycholinguistic Database (Wilson, 1988) with mid-range values of frequency, concreteness, meaningfulness, and word length. The words were shown in bolded black in 36-point Times New Roman font in the center of the screen. The remember cue was a green " $R$ " and the forget cue was a red "F". Both were shown in 72-point bolded Times New Roman font in the center of the screen.

Design Experiments $1 \mathrm{~A}$ and $1 \mathrm{~B}$ constituted a 2 (Test Type: item recognition vs. associative recognition) $\times 2$ (Cue: remember vs. forget) $\times 2$ (Test Probe: old vs. new) incomplete within-subjects design. The design was necessarily incomplete because the cue manipulation could not be varied for new (unstudied) item recognition test probes.

Procedure Experiments $1 \mathrm{~A}$ and $1 \mathrm{~B}$ were run consecutively and differed only in terms of the initial presentation time of the pairs at study. The study list consisted of a total of 76 pairs of which 72 were critical pairs. The first and last two pairs were included to buffer primacy and recency effects and were not tested. Participants initiated the study list when they were ready to do so. In Experiment $1 \mathrm{~A}$, each pair was shown in the center of the screen for $2 \mathrm{~s}$ before being replaced by the $\mathrm{R}$ or $\mathrm{F}$ cue. In Experiment $1 \mathrm{~B}$ the pair was shown for $1 \mathrm{~s}$ before the presentation of the cue. The cue was shown for $3 \mathrm{~s}$. Of the 72 critical word pairs, 36 were followed by the R cue and 36 by the $\mathrm{F}$ cue. The order of the critical word pairs was randomized with a different order for each participant. The pairs that received the $\mathrm{R}$ or $\mathrm{F}$ cue were counterbalanced across participants in each experiment.

After the study list, the participants received instructions on how to complete the test phase. It was emphasized that old responses should be made to any single words or any word pairs that were presented in the study list regardless of whether they were followed by an R or an F cue. Participants initiated the test list when they were ready. The test list contained 24 single old items, each from a different study pair, and 24 single new items. Twelve of the 24 old items were from pairs that were cued to be remembered and 12 were from pairs cued to be forgotten. The test list also contained 24 intact old pairs and 24 rearranged new pairs for the associative recognition tests. Half of the intact pairs were cued to be remembered and half were cued to be forgotten. Similarly, half of the rearranged test pairs were composed of items from R-cued study pairs and half were composed of items from F-cued pairs. No word appeared in more than one test probe. Test order was random with a different order for each participant. The participants indicated whether the single word or the word pair was old or new by pressing the "/" or the " $\mathrm{z}$ " key on the keyboard. The test phase was subject-paced with the next test probe appearing after a response.

\section{Results}

The mean proportions of old responses for R-cued and F-cued old (hits) and new (false alarms) item and associative recognition tests are presented in Table 1. The .05 level of significance was used to interpret all statistical outcomes.

Item recognition $\mathrm{A}$ directed forgetting effect was observed in both experiments. Paired sample t-tests showed that the hit rate for R-cued items was greater than the hit rate for F-cued items in Experiment 1A, $t(23)=3.174, p=.004$, and Experiment 1B, $t(23)=2.312, p=.03$. A 2 (Cue: R vs. F) $\times 2$ (Experiment: $1 \mathrm{~A}$ vs. 1B) mixed factor analysis of variance (ANOVA) was carried out on the hit rates to compare performance between the two experiments. The hit rate was greater for R-compared to Fcued items, $F(1,46)=14.27, M S E=0.016$, eta ${ }^{2}=0.237$, $p<.001$, and greater with the 2-s presentation time of Experiment $1 \mathrm{~A}$ than the 1 -s presentation time of Experiment $1 \mathrm{~B}, F(1$, $46)=11.89, M S E=.030$, eta $^{2}=0.205, p<0.001$. The interaction between Cue and Experiment was not significant, $F(1,46)$ $<1$. The directed forgetting effect (hit rate for R-cued items minus hit rate for F-cued items) was the same in Experiment $1 \mathrm{~A}(\mathrm{M}=.10, \mathrm{SE}=.03)$ and Experiment $1 \mathrm{~B}(\mathrm{M}=.10, \mathrm{SE}=.03)$. 
Table 1 Mean hit rates, false alarm rates, and corrected recognition scores for remember and forget cued item and associative recognition for the 2-s and 1-s presentation durations of Experiments $1 \mathrm{~A}$ and $1 \mathrm{~B}$

\begin{tabular}{|c|c|c|c|c|c|}
\hline \multirow[t]{2}{*}{ Duration } & \multirow[t]{2}{*}{ Cue } & \multicolumn{2}{|c|}{ Item recognition } & \multicolumn{2}{|c|}{ Associative recognition } \\
\hline & & M & $\mathrm{SE}$ & M & $\mathrm{SE}$ \\
\hline \multicolumn{6}{|l|}{ Hit rates } \\
\hline \multirow[t]{2}{*}{$2 \mathrm{~s}$} & Remember & .64 & .03 & .63 & .04 \\
\hline & Forget & .54 & .03 & .52 & .03 \\
\hline \multirow[t]{2}{*}{$1 \mathrm{~s}$} & Remember & .52 & .03 & .64 & .03 \\
\hline & Forget & .42 & .03 & .53 & .03 \\
\hline \multicolumn{6}{|c|}{ False alarm rates } \\
\hline \multirow[t]{2}{*}{$2 \mathrm{~s}$} & Remember & .18 & .03 & .36 & .04 \\
\hline & Forget & & & .37 & .04 \\
\hline \multirow[t]{2}{*}{$1 \mathrm{~s}$} & Remember & .24 & .03 & .43 & .04 \\
\hline & Forget & & & .42 & .04 \\
\hline \multicolumn{6}{|c|}{ Corrected recognition } \\
\hline \multirow[t]{2}{*}{$2 \mathrm{~s}$} & Remember & .46 & .05 & .27 & .06 \\
\hline & Forget & .36 & .03 & .16 & .06 \\
\hline \multirow[t]{2}{*}{$1 \mathrm{~s}$} & Remember & .28 & .05 & .22 & .05 \\
\hline & Forget & .19 & .03 & .11 & .05 \\
\hline
\end{tabular}

Note: New items in the item recognition tests were not on the study list and therefore were not cued. For associative recognition, remember and forget false alarm rates refer to the study condition of the items in the rearranged pairs

The difference in false alarm rates between the two experiments was not significant, $t(46)=1.30, p=.199$.

Associative recognition A 2 (Test Pair: intact vs. rearranged) $\times 2$ (Cue: R vs. F) $\times 2$ (Experiment: 1A vs. 1B) mixed factor ANOVA was carried out on the proportion of old responses. The proportion of old responses was significantly greater for intact than rearranged pairs, $F(1,46)=58.15, M S E=0.030$, $\mathrm{eta}^{2}=0.558, p<0.001$, and greater for R-cued than $\mathrm{F}$-cued pairs, $F(1,46)=5.495, M S E=0.027$, eta ${ }^{2}=0.107, p=.023$, but did not differ reliably between experiments, $F(1,46)=$ $1.607, M S E=.036$, eta $^{2}=.034, p=.211$. The significant main effects of Test Pair and Cue were qualified by their interaction, $F(1,46)=4.77, M S E=0.029$, eta $^{2}=.094, p=.034$. The difference in hit rates between $\mathrm{R}$-cued $(\mathrm{M}=.64, \mathrm{SE}=.02)$ and $\mathrm{F}$-cued $(\mathrm{M}=.53, \mathrm{SE}=.02)$ intact pairs was greater than the difference in false alarm rates between $\mathrm{R}$-cued $(\mathrm{M}=.39$, $\mathrm{SE}=.03)$ and $\mathrm{F}$-cued $(\mathrm{M}=.39, \mathrm{SE}=.03)$ rearranged pairs. The interactions between Test Pair and Experiment, $F(1,46)=$ 1.10, Cue and Experiment, $F(1,46)<1$, and Test Pair, Cue, and Experiment, $F(1,46)<1$, did not approach significance.

To examine discrimination performance for $\mathrm{R}$ - and F-cued pairs, corrected recognition scores (hit rate minus the associated false alarm rate) were calculated. These means are also shown in Table 1. Corrected recognition was compared in a 2 (Cue: R vs. F) $\times 2$ (Experiment: $1 \mathrm{~A}$ vs. 1B) mixed factor ANOVA. Associative recognition was significantly greater for R- compared to F-cued pairs, $F(1,46)=4.77, M S E=.028$, eta $^{2}=.094, p=.034$, demonstrating a directed forgetting effect for associative discrimination. The main effect of Experiment, $F(1,46)=$ $1.10, M S E=.059$, eta $^{2}=.023, p=.299$, and the Cue by Experiment interaction, $F(1,46)<1$, did not approach significance. Thus, the difference in presentation time between Experiments 1A and 1B did not influence associative recognition performance. The magnitude of the directed forgetting effect based on the difference between R-cued and F-cued corrected recognition scores was also similar for Experiment $1 \mathrm{~A}(\mathrm{M}=.11, \mathrm{SE}=.07)$ and $\mathrm{Ex}-$ periment $1 \mathrm{~B}(\mathrm{M}=.10, \mathrm{SE}=.07)$.

As noted in the above analysis, the corrected recognition scores were reduced for F-cued compared to R-cued pairs, illustrating the effect of directed forgetting. One-sample t-tests showed that the F-cued corrected recognition scores were above chance in both Experiment $1 \mathrm{~A}, t(23)=3.46, p=.002$, and Experiment 1B, $t(23)=2.38, p=.026$, demonstrating the incidental encoding of associative information for to-beforgotten pairs.

Item versus associative recognition To compare discrimination for item and associative recognition, a 2 (Test Type: item vs. associative recognition) $\times 2$ (Cue: R vs. F) $\times 2$ (Experiment: $1 \mathrm{~A}$ vs. 1B) mixed factor ANOVA was carried out on corrected recognition scores. Discrimination was greater for item compared to associative recognition, $F(1,46)=21.66, M S E=$ .039 eta $^{2}=.320, p<.001$, for R-cued than F-cued information, $F(1,46)=15.45, M S E=.033$ eta $^{2}=.251, p<.001$, and for the 2-s presentation time in Experiment 1A compared to the 1-s duration of Experiment 1B, $F(1,46)=7.42, M S E=.028$, eta $^{2}$ $=.139, p=.009$. The interaction between Test Type and Experiment was also reliable, $F(1,46)=4.86, M S E=.039$, eta $^{2}=$ $.096, p=.033$; discrimination decreased for item recognition with the decrease in presentation time from Experiment 1A to 1B but not for associative recognition. The Cue $\times$ Experiment, Cue $\times$ Test Type, and Cue $\times$ Test Type $\times$ Experiment interactions did not approach significance (all $F s<1$ ).

The results of Experiment 1A and 1B demonstrate a directed forgetting effect for both item and associative recognition. Discrimination for item recognition was also reduced with the decrease in presentation time before the cue between experiments. In contrast, associative recognition discrimination did not differ with pre-cue duration.

According to the differential rehearsal account of item-based directed forgetting, participants should hold the two items in short-term or working memory until the cue is presented. Given an R cue, participants should then generate and rehearse an associative relation for the pair. When the cue is $\mathrm{F}$, the two words should be forgotten or at least dropped from mind. Therefore, in the case of F-cued pairs, the 
individual items should derive some benefit of maintenance rehearsal but little to no associative information should be encoded. The results of Experiment 1A and 1B are largely consistent with this differential rehearsal account. Memory was better for R-cued compared to F-cued items and pairs reflecting the benefits of increased attention and rehearsal. Both item and associative information benefited to a similar extent from the R-cue instruction because emphasizing the encoding of associative information also aids the encoding of item information (Hockley \& Cristi, 1996). Discrimination was also greater for individual items than for pairs possibly reflecting the initial attention to, and maintenance rehearsal of, the items.

The results also showed, however, that participants did encode relational information to support above chance associative recognition of F-cued pairs, although not to the same extent as for R-cued pairs. This result is consistent with previous demonstrations of the incidental encoding of associative information (Jou, 2010; Hockley \& Cristi, 1996). The encoding of associative information when memory for the items and their pairing is not required suggests that participants may spontaneously encode relational information, at least for a reasonable proportion of the pairs. In contrast to item recognition, associative recognition did not benefit from a longer presentation duration prior to the cue further suggesting that the spontaneous encoding of associations between random words occurs early in the processing of the two items. Experiment 2 was designed to make such spontaneous encoding of relational information easier and more likely by presenting pairs with pre-existing associations.

\section{Experiment 2}

Experiments 1A and 1B showed that participants encoded associations between random pairs of words even when instructed not to do so. In Experiment 2, half of the study pairs were two members (or more properly, lexemes) of compound words (e.g., check list, needle point) and half were unrelated word pairs that were rearranged members of compound words. At test, the rearranged pairs were also either compound word pairs (e.g., check point) or unrelated word pairs. Ahmad and Hockley (2014) showed that in yes-no associative recognition, the hit and false alarm rates are greater for compound word (CW) pairs compared to non-compound word (NCW) pairs without any difference in overall discrimination performance. Ahmad and Hockley also showed that the encoded $\mathrm{CW}$ pairs were unitized and that unitization provides a basis to use familiarity to make associative recognition decisions in the same way that familiarity can be used in making recognition judgments for individual words (see also Quamme, Yonelinas \& Norman, 2007). Ahmad, Fernandes, and Hockley (2015) went on to show that the unitization of compound word pair associations can also reduce older adults' associative memory deficit (Naveh-Benjamin, 2000) by aiding encoding of associations between items and providing the opportunity for familiarity-based decisions that are known to be spared in older adults.

Experiment 2 was designed to determine if the incidental encoding of relational information is increased when it is made easier due to the pre-experimental associations inherent in $\mathrm{CW}$ pairs. It was predicted that the directed forgetting effect would be reduced for $\mathrm{CW}$ pairs compared to $\mathrm{NCW}$ pairs due to the ease of encoding associations between the members of CW pairs, and the ability to make familiarity-based associative recognition decisions for these unitized word pairs.

\section{Method}

Participants A total of 28 students were tested. Two participant data files were excluded because they showed overall chance performance for both $\mathrm{CW}$ and $\mathrm{NCW}$ pairs that were followed by remember cues.

Apparatus and materials The apparatus was the same as in previous experiments. For the stimuli, from a list of 180 compound words provided by Jones (2005), $60 \mathrm{CW}$ pairs and $60 \mathrm{NCW}$ pairs were constructed. The first member of the parent $\mathrm{CW}$ pair was paired with second member of another $\mathrm{CW}$ pair to create a rearranged $\mathrm{CW}$ pair. To create the NCW pairs, the left word from one $\mathrm{CW}$ pair was paired with the right word from an unrelated $\mathrm{CW}$ pair to form a quasi-random word pair. This was done to equate the individual words in the $\mathrm{CW}$ and NCW pairs. Similar to Ahmad and Hockley (2014), two sets of pairs were constructed to counterbalance the components of the $\mathrm{CW}$ and $\mathrm{NCW}$ pairs across participants.

Procedure The study list consisted of $60 \mathrm{CW}$ and $60 \mathrm{NCW}$ pairs. For both word pair types, 30 word pairs were followed by a remember cue and 30 pairs were followed by a forget cue. In addition, there were two pairs (one $\mathrm{R}$ and one $\mathrm{F}$ cued) at the beginning and two at end of the list that served as primacy and recency buffers and were not tested. For R-cued pairs, participants were instructed to form an association between the word pair in order to better remember the pair for the recognition test. In addition, they were told to try making a sentence or image that involved both words in the word pair to make it easier for them to remember the word pairs. Each pair was presented for $2 \mathrm{~s}$ which was followed by an R or F cue presented for $3 \mathrm{~s}$. The order of presentation was random for each participant.

After the study phase was completed, for approximately $1 \mathrm{~m}$ duration, the experimenter presented the test instructions to the participants. They were informed of the difference between old (intact) and new (rearranged) test pairs and were instructed to press the "/" and " $\mathrm{z}$ " keys for old and new 
judgments, respectively. They were also told to make their recognition decisions regardless if the pairs were cued to be remembered or forgotten during the study phase.

During the test phase, each trial began with a test pair displayed on the center of the screen in the same format as the study pairs. Test pairs remained on the screen until the participants responded. There were 20 intact and 20 rearranged $\mathrm{CW}$ pairs and 20 intact and 20 rearranged $\mathrm{NCW}$ pairs presented in a different random order for each participant. Half of the intact pairs had been presented with a remember cue. Similarly for the rearranged pairs, half were constructed from pairs that had been presented with an $\mathrm{R}$ cue and half from pairs followed by an $\mathrm{F}$ cue.

\section{Results and discussion}

The mean hit and false alarm rates for R-cued and F-cued intact and rearranged $\mathrm{CW}$ and $\mathrm{NCW}$ pairs are presented in Table 2. A 2 (Test Probe: Old vs. New) $\times 2$ (Cue: R vs. F cue) $\times 2$ (Pair Type: $\mathrm{CW}$ vs. NCW) repeated measures ANOVA showed that the proportion of old responses was greater for intact than rearranged pairs, $F(1,25)=131.79$, $M S E=0.023$, eta $^{2}=0.841, p<0.001$, greater for R-cued than F-cued pairs, $F(1,25)=43.23, M S E=0.036$, eta ${ }^{2}=0.634$, $p<0.001$, and greater for CW than NCW pairs, $F(1,25)=$ 29.68, MSE $=0.044$, eta $^{2}=0.543, p<0.001$. These main effects were qualified by two interactions. The Test Probe $\times$ Cue interaction was significant, $F(1,25)=31.57, M S E=$ 0.022, eta $^{2}=0.558, p<0.001$. The difference in hit rates between $\mathrm{R}$ and $\mathrm{F}$ cued pairs was much greater than the difference in false alarm rates. The interaction between Test Probe and Pair Type was also significant, $F(1,25)=7.70, M S E=$ 0.014, eta $^{2}=0.235, p=.01$, as the difference between CW and NCW pairs was greater for hits (.20) than for false alarms (.12). The interactions between Cue and Pair Type, $F(1,25)<$ 1 , and Cue, Pair Type, and Test Pair, $F(1,25)=2.923, M S E=$ 0.014, eta $^{2}=0.105, p=.10$, were not significant.

Mean corrected recognition scores for R-cued and F-cued $\mathrm{CW}$ and NCW pairs are also shown in Table 2. A 2 (Cue: R vs. F) $\times 2$ (Pair Type: $\mathrm{CW}$ vs. NCW) repeated measures ANOVA based on these scores confirmed that associative discrimination was greater for $\mathrm{R}(\mathrm{M}=.36, \mathrm{SE}=.03)$ than $\mathrm{F}$-cued $(\mathrm{M}=.12$, $\mathrm{SE}$ $=.03)$ pairs, $F(1,25)=31.57, M S E=0.045$, eta $^{2}=0.558$, $p<.001$. Discrimination was also greater for $\mathrm{CW}(\mathrm{M}=.28$, $\mathrm{SE}=.03)$ than $\mathrm{NCW}(\mathrm{M}=.19, \mathrm{SE}=.03)$ pairs, $F(1,25)=7.70$, $M S E=0.028$, eta $^{2}=0.235, p=.01$. The interaction between Cue and Pair type approached significance, $F(1,25)=2.92$, $M S E=0.028$, eta $^{2}=0.105, p=.10$. Planned comparisons based on paired t-tests showed that there was no difference in corrected recognition scores for $\mathrm{CW}$ and $\mathrm{NCW}$ R-cued pairs, $t(25)=0.874, p=.39$, but corrected recognition was greater for F-cued CW compared to NCW pairs, $t(25)=2.82, p=.009$. Therefore, as predicted, the directed forgetting effect was
Table 2 Mean hit rates, false alarm rates, and corrected recognition scores for remember and forget compound and non-compound word pairs in Experiment 2

\begin{tabular}{|c|c|c|c|c|}
\hline \multirow[t]{2}{*}{ Cue } & \multicolumn{2}{|c|}{ Compound word pairs } & \multicolumn{2}{|c|}{ Non-compound word pairs } \\
\hline & M & SE & M & $\mathrm{SE}$ \\
\hline \multicolumn{5}{|l|}{ Hit rates } \\
\hline Remember & .77 & .02 & .62 & .04 \\
\hline Forget & .53 & .05 & .28 & .04 \\
\hline \multicolumn{5}{|c|}{ False alarm rates } \\
\hline Remember & .40 & .04 & .28 & .04 \\
\hline Forget & .34 & .04 & .23 & .03 \\
\hline \multicolumn{5}{|l|}{$\begin{array}{l}\text { Corrected } \\
\quad \text { recognition }\end{array}$} \\
\hline Remember & .37 & .04 & .34 & .03 \\
\hline Forget & .20 & .05 & .05 & .04 \\
\hline
\end{tabular}

Note: Remember and forget false alarm rates refer to the study condition of the items in the rearranged pairs

greater for NCW pairs $(\mathrm{M}=.29, \mathrm{SE}=.04)$ than for $\mathrm{CW}$ pairs $(\mathrm{M}=.17, \mathrm{SE}=.06), t(25)=1.71, p=.048$. Discrimination performance was significantly greater than chance for $\mathrm{F}$-cued $\mathrm{CW}$ pairs $(\mathrm{M}=.20, \mathrm{SE}=.05), t(25)=4.40, p<.01$, but not for F-cued NCW pairs $(\mathrm{M}=.05, \mathrm{SE}=.04), t(25)=1.42, p=.17$.

The results for the R-cued CW and NCW pairs replicated the compound word effect for intentionally studied word pairs described by Ahmad and Hockley (2014); the hit and false alarm rates were greater for R-cued $\mathrm{CW}$ word pairs compared to R-cued NCW pairs but there was no significant difference in discrimination between the pair types. There was also a directed forgetting effect, as hit rates were greater for both R-cued CW and NCW pairs compared to F-cued pairs. Importantly, and as predicted, the directed forgetting effect was reduced for $\mathrm{CW}$ compared to NCW pairs. Participants were more likely to spontaneously encode the pre-experimental associations inherent in $\mathrm{CW}$ pairs which produced a discrimination advantage for the F-cued $\mathrm{CW}$ pairs compared to the F-cued NCW pairs. It is also likely that CW pairs, once unitized, also benefited from maintenance rehearsal prior to the cue in the same way that single items benefited in Experiments 1A and 1B.

In contrast to the $\mathrm{F}$-cued random word pairs of Experiments $1 \mathrm{~A}$ and $1 \mathrm{~B}$, and the $\mathrm{F}$-cued $\mathrm{CW}$ pairs of Experiment 2, the modest level of associative recognition for the F-cued NCW pairs of Experiment 2 did not differ significantly from chance. As the construction of the random word pairs in Experiment 2 was constrained by the necessity of re-pairing unrelated components of compound words, these word pairs may have been more difficult to associate than the word pairs used in Experiments 1A and 1B. Previous research has shown that associative recognition varies depending on the nature of the words in studied pairs. For example, associative recognition accuracy is greater for random pairs of concrete compared to abstract nouns (Hockley, 1994). It is also possible that participants 
may have paid more attention to whether or not the word pairs of Experiment 2 formed compound words and were consequently less likely to form relations between the random word pairs. In either event, this result demonstrates that there are limits to the incidental encoding and subsequent discrimination of associative information.

\section{General discussion}

The results of the present experiments provide a further demonstration of the incidental or unintended encoding of associative information. Previously, Hockley and Cristi (1996) and Jou (2010) have shown that participants do encode associative information even when they are instructed to emphasize the encoding of item information, although not to the same extent as when they focus on making associations between unrelated word pairs. Bancroft et al. (2013) further demonstrated the incidental encoding of associative information for F-cued pairs with the item-based directed forgetting procedure. Bancroft et al. presented random word pairs for $3 \mathrm{~s}$ before the cue was given. Similar results were found in Experiments 1A and 1B when the initial presentation duration was reduced to $2 \mathrm{~s}$ and $1 \mathrm{~s}$, respectively. These experiments provide an especially convincing demonstration of the incidental or spontaneous encoding of associative information because, unlike the procedures of Hockley and Cristi (1996) and Jou (2010), participants were instructed to forget both the items and the pairs when the F cue was presented. Therefore, participants should have no incentive to try to generate associations between the two words of the pairs.

It is also important to note that the incidental encoding of associative information is not obligatory. In contrast to the random word pairs in Experiments $1 \mathrm{~A}$ and $1 \mathrm{~B}$, the very modest level of recognition performance for F-cued NCW pairs in Experiment 2 was not reliably greater than chance. As the nature of the random words in these experiments was different, incidental encoding of relations between items may depend on the ease of generating associations between different types of items. And, as the $\mathrm{CW}$ condition of Experiment 2 showed, pre-experimental associations between the items makes the incidental encoding of associative information much more likely.

The incidental encoding of associative information appears to have an all-or-none rather than a graded or gradual quality. As the comparison between Experiments 1A and 1B showed, decreasing the initial presentation time of the pair reduced discrimination for both R-cued and F-cued item recognition, but did not affect R-cued or F-cued associative recognition. The encoding of item information benefited from the increased maintenance of, or attention paid to, the two words of each pair prior to the presentation of the cue whereas associative recognition did not. Participants may encode a relation between the words of an F-cued pair if one comes spontaneously to mind, but the probability of such associations do not increase with time, at least over the intervals tested here. As such, the incidental or spontaneous encoding of associative information between items may be similar to the one-shot hypothesis of context storage proposed by Malmberg and Shiffrin (2005).

It is interesting to note that whereas presentation time before the cue, at least over the first few seconds, was not observed to influence the encoding of associative information, Bancroft et al. (2013) found that the duration after the cue (2-6 s) led to increased associative recognition. It appears that the duration before and after the cue may have different consequences for associative memory. Increasing the duration before the cue improves item recognition, presumably due to the benefits of maintenance rehearsal, but does not benefit associative recognition. Increasing the duration after the cue improves both item and associative recognition, and surprisingly, this benefit is seen to a similar extent for F-cued pairs as well as R-cued pairs. As Bancroft et al. speculated, the general benefit of post cue duration for F-cued item and associative information is an ironic memorial benefit for the information that one attempts to forget, and may be a consequence of inefficient or counterproductive forgetting strategies.

The results of the present experiments also replicate and extend several additional findings that have been observed in tests of associative recognition. For one, in both Experiments $1 \mathrm{~A}$ and $1 \mathrm{~B}$ the effect of directed forgetting was seen almost exclusively in the hit rates of intact pairs with little difference in the false alarm rates of rearranged $\mathrm{R}$ and F-cued pairs. This pattern is consistent with results of previous studies that have manipulated within-list pair strength in tests of associative recognition. Kelley and Wixted (2001) showed that when some pairs have longer presentation times or are presented more often than other pairs at study, subsequent tests of associative recognition show the expected increase in hit rates, but no difference in false-alarm rates between rearranged test pairs composed of items from strong and weak pairs (see also Cleary, Curran, \& Greene, 2001; Verde \& Rotello, 2004; Xu \& Malmberg, 2007). Kelley and Wixted (2001) suggested that strengthening study pairs increases both the familiarity of the individual items and the likelihood of retrieving the original studied pair. These factors work together to increase the hit rate for intact pairs, but act in opposition for rearranged pairs. No difference is seen in the false-alarm rates of strong and weak rearranged pairs when the increase in item familiarity is offset by the increase in the probability of correctly recalling the study pair (i.e., recall-to-reject, Rotello \& Heit, 2000).

Although false alarm rates for associative recognition were similar for R- and F-cued rearranged pairs in Experiments $1 \mathrm{~A}$ and $1 \mathrm{~B}$, they were greater for $\mathrm{CW}$ than for $\mathrm{NCW}$ rearranged pairs in Experiment 2. In terms of Kelley and Wixted's (2001) account of associative recognition, the additional pre-experimental familiarity of the $\mathrm{CW}$ rearranged pairs may have offset the balance between 
increases in item familiarity and associative recall leading to an increased false alarm rate.

The results of the R-cued pairs of Experiment 2 also replicated and extended the concordant compound pair effect documented by Ahmad and Hockley (2014; see also Ahmad et al., 2015). The hit and false alarm rates were greater for $\mathrm{CW}$ pairs compared to NCW pairs with no difference in overall discrimination performance. Ahmad and Hockley accounted for this effect in terms of the greater familiarity of the $\mathrm{CW}$ pairs.

The discrimination of F-cued CW pairs, on the other hand, was greater than that of F-cued NCW pairs.

Ahmad et al. (2015) observed that older adults, like younger adults, also show higher hit and false alarm rates for $\mathrm{CW}$ compared to NCW word pairs. In contrast to younger adults, however, older adults show a discrimination advantage for $\mathrm{CW}$ pairs. Ahmad et al. argued that the ease of making associations between the members of $\mathrm{CW}$ pairs, and the ability to make familiarity-based associative recognition decisions for these unitized word pairs, served to attenuate the age-related associative deficit typically observed for older adults (cf. Naveh-Benjamin, 2000). Interestingly, the pattern of results for R-cued and F-cued CW and NCW pairs seen in Experiment 2 mimicked the pattern of associative recognition results observed for young and older adults by Ahmad et al. (2015). The incidental encoding of associative information for F-cued pairs benefits from the ease of encoding relations between members of $\mathrm{CW}$ pairs in the same way that older adults benefited from the pre-experimental associations afforded by $\mathrm{CW}$ pairs. The strategic encoding and rehearsal strategies available to young, but not older, adults can overcome the advantage of $\mathrm{CW}$ pairs when they are employed for R-cued NCW pairs,

As the present experiments show, the item-based directed forgetting procedure is an ideal way to assess the incidental encoding of associative information. Future research could compare the spontaneous encoding of associations for different types of stimuli in addition to word pairs. One important question to address is whether incidental associations are encoded as frequently or as effectively for between-domain information (e.g., word-face pairs) as within-domain information (cf. domain dichotomy theory; Bastin, Linden, Schnakers, Montaldi, \& Mayes, 2010).

In summary, the present results demonstrate that young adults often encode associations between random pairs of words even when instructed not to do so. These associations appear to be spontaneous and do not increase over short periods of time. Pre-experimental associations, as in the case of the components of compound words, increase the likelihood of encoding such associations. These spontaneous associations, though, are not as effective in supporting later associative recognition as the associations that young adults deliberately try to remember.
Author Notes This study was supported by a Discovery Grant from the National Engineering and Research Council of Canada to the first author Correspondence can be directed to W.E. Hockley, Department of Psychology, Wilfrid Laurier University, Waterloo, ON, N2L 3C5, Canada, or electronically to whockley@wlu.ca.

\section{References}

Ahmad, F. N., Fernandes, M., \& Hockley, W. E. (2015). Improving associative memory in older adults with unitization. Aging, Neuropsychology, and Cognition, 22, 452-472.

Ahmad, F. N., \& Hockley, W. E. (2014). The role of familiarity in associative recognition of unitized compound word pairs. Quarterly Journal of Experimental Psychology, 12, 2301-2324.

Bancroft, T. D., Hockley, W. E., \& Farquhar, R. (2013). The longer we have to forget the more we remember: The ironic effect of post-cue duration in item-based directed forgetting. Journal of Experimental Psychology: Learning, Memory, and Cognition, 39, 691-699.

Basden, B., \& Basden, D. (1998). Directed forgetting: A contrast of methods and interpretations. In J. Golding \& C. M. MacLeod (Eds.), Intentional forgetting: Interdisciplinary approaches (pp. 139-172). Mahwah: Erlbaum.

Basden, B., Basden, D., \& Gargano, G. (1993). Directed forgetting in implicit and explicit memory tasks: A comparison of methods. Journal of Experimental Psychology: Learning, Memory, and Cognition, 19, 603-616.

Bastin, C., Linden, M., Schnakers, C., Montaldi, D., \& Mayes, A. R. (2010). The contribution of familiarity to within- and betweendomain associative recognition memory: Use of a modified remember/know procedure. European Journal of Cognitive Psychology, 22, 922-943.

Bjork, R. A. (1989). Retrieval inhibition as an adaptive mechanism in human memory. In H. L. Roediger III \& F. I. M. Craik (Eds.), Varieties of memory and consciousness: Essays in honour of Endel Tulving (pp. 309-330). Hillsdale: Erlbaum.

Cleary, A. M., Curran, T., \& Greene, R. L. (2001). Memory for detail in item versus associative recognition. Memory \& Cognition, 29, 413-423.

Glenberg, A., \& Adams, F. (1978). Type I rehearsal and recognition. Verbal Learning and Verbal Behavior, 17, 455-463.

Godden, D. R., \& Baddeley, A. D. (1975). Context-dependent memory in two natural environments: On land and underwater. British Journal of Psychology, 66, 325-331.

Golding, J. M., \& Long, D. L. (1998). There's more to intentional forgetting than directed forgetting: An integrative review. In J. M. Golding \& C. M. MacLeod (Eds.), Intentional forgetting: Interdisciplinary approaches (pp. 59-102). Mahwah: Erlbaum.

Hanczakowski, M., Pasek, T., \& Zawadzka, K. (2012). Contextdependent impairment of recollection in list-method directed forgetting. Memory, 20, 758-770.

Hockley, W. E. (1994). Reflections of the mirror effect for item and associative recognition. Memory \& Cognition, 22, 713-722.

Hockley, W. E. (2008). The effects of environmental context on recognition memory and claims of remembering. Journal of Experimental Psychology: Learning, Memory, and Cognition, 34, 1412-1429.

Hockley, W. E., \& Cristi, C. (1996). Tests of encoding tradeoffs between item and associative information. Memory \& Cognition, 24, 202-216.

Humphreys, M. S. (1976). Relational information and the context effect in recognition memory. Memory \& Cognition, 4, 221-232.

Johnson, H. M. (1994). Processes of successful intentional forgetting. Psychological Bulletin, 116, 274-292. 
Jones, T. C. (2005). Study repetition and the rejection of conjunction lures. Memory, 13, 499-515.

Jou, J. (2010). Can associative information be strategically separated from item information in word-pair recognition? Psychonomic Bulletin and Review, 17, 778-783.

Kelley, R., \& Wixted, J. T. (2001). On the nature of associative information in recognition memory. Journal of Experimental Psychology: Learning, Memory, and Cognition, 27, 701-722.

MacLeod, C. M. (1998). Directed forgetting. In J. Golding \& C. M. MacLeod (Eds.), Intentional forgetting: Interdisciplinary approaches (pp. 139-172). Mahwah: Erlbaum.

Malmberg, K. J., \& Shiffrin, R. M. (2005). The "oneshot" hypothesis for context storage. Journal of Experimental Psychology: Learning, Memory, and Cognition, 31, 322-336.

Murdock, B. B., JR. (1974). Human memory: Theory and data. Hillsdale, NJ: Erlbaum.

Naveh-Benjamin, M. (2000). Adult age differences in memory performance: Tests of an associative deficit hypothesis. Journal of Experimental Psychology: Learning, Memory, and Cognition, 26, $1170-1187$.
Naveh-Benjamin, M., \& Jonides, J. (1984). Cognitive load and maintenance rehearsal. Journal of Verbal Learning and Verbal Behavior, 23, 494-507.

Quamme, J. R., Yonelinas, A. P., \& Norman, K. A. (2007). Effect of unitization on associative recognition in amnesia. Hippocampus, 17, 192-200.

Rotello, C. M., \& Heit, E. (2000). Associative recognition: A case of recall-to-reject processing. Memory \& Cognition, 28, 907-922.

Verde, M. F., \& Rotello, C. M. (2004). Strong memories obscure weak memories in associative recognition. Psychonomic Bulletin \& Review, 11, 1062-1066.

Wilson, M. D. (1988). The MRC Psycholinguistic Database: Machine Readable Dictionary, Version 2. Behavioural Research Methods, Instruments, \& Computers, 20, 6-11.

Woodward, A. E., Jr., Bjork, R. A., \& Jongeward, R. H., Jr. (1973). Recall and recognition as a function of primary rehearsal. Journal of Verbal Learning and Verbal Behavior, 12, 608-617.

Xu, J., \& Malmberg, K. J. (2007). Modeling the effects of verbal and nonverbal pair strength on associative recognition. Memory \& Cognition, 35, 526-544. 